\title{
THE AREA OF THE COMPLEMENT OF A CONFORMALLY RIGID DOMAIN
}

\author{
MARIUS OVERHOLT
}

(Communicated by Irwin Kra)

\begin{abstract}
A hyperbolic subdomain $D$ of $\hat{\mathbf{C}}$ is said to be (conformally) rigid if any conformal map from $D$ into $\hat{\mathbf{C}}$ is either a Möbius transformation, or has Schwarzian norm larger than a positive constant depending on $D$ only. We show that the complement of a conformally rigid domain has zero area.
\end{abstract}

1. Introduction. Let $D$ be a hyperbolic subdomain (not necessarily simply connected) of $\hat{\mathbf{C}}$; i.e., $D$ has the unit disk $\mathbf{D}$ as its universal covering surface. Then $D$ carries a metric of constant negative curvature, called the Poincaré metric on $D$. We shall denote its density by $\rho_{D}(z)$. It is inherited from the Poincaré density

$$
\rho_{\mathbf{D}}(z)=\left(1-|z|^{2}\right)^{-1}
$$

of the unit disk, via the covering projection. The hyperbolic sup-norm of weight -2 on $D$ is given by

$$
\|\phi\|_{D}=\sup _{z \in D}|\phi(z)| \rho_{D}(z)^{-2}
$$

Further, if $f: L \rightarrow \hat{\mathbf{C}}$ is a locally univalent meromorphic function, then the Schwarzian derivative

$$
S(f)=\left(f^{\prime \prime} / f^{\prime}\right)^{\prime}-\frac{1}{2}\left(f^{\prime \prime} / f^{\prime}\right)^{2}
$$

is a holomorphic function on $D$, and we may form the Schwarzian norm $\|S(f)\|_{D}$ of $f$ on $D$. It may be infinite, of course.

The following definition is due to Thurston [5].

Definition (ThURSTON). A hyperbolic domain $D \subseteq \hat{\mathbf{C}}$ is (conformally) rigid if there exists a constant $s>0$ such that if $f: D \rightarrow \hat{\mathbf{C}}$ is conformal and $\|S(f)\|_{D} \leq$ $s$, then $f$ is a Möbius transformation.

In this definition, and elsewhere in this paper, conformal is to mean holomorphic and globally injective.

In [5] Thurston proved the existence of rigid domains by construction. His examples are complements of quasiarcs, and so the complements of these rigid domains automatically have zero area. Astala [1] gives an alternative proof for the existence of rigid domains. His examples are contained among those of Thurston.

Received by the editors December 1, 1986 and, in revised form, May 5, 1987.

1980 Mathematics Subject Classification (1985 Revision). Primary 30C35.

Key words and phrases. Conformal map, Schwarzian derivative, rigid domain.

This research was supported by the Norwegian Research Council for Science and the Humanities, and a Rackham Predoctoral Fellowship.

This research forms part of the author's Ph.D. thesis at the University of Michigan under the direction of Professor Peter L. Duren. 
2. Complements of rigid domains. In the following, $m(A)$ will always denote the Lebesgue planar measure of a set $A$. We shall need the following theorem due to Nguyen Xuan Uy [4].

THEOREM (NGUYEN). If $\Gamma$ is compact in $\mathbf{C}$, with $m(\Gamma)>0$, then there exists a nonconstant bounded holomorphic Lipschitz function on $\hat{\mathbf{C}} \backslash \Gamma$.

In addition, we shall need the following result due to Beardon and Gehring [2].

THEOREM (BEARDON AND GEHRING). If $D$ is a hyperbolic subdomain of $\hat{\mathbf{C}}$, then

$$
|S(f, z)| \leq 12 \rho_{D}(z)^{2}
$$

in $D$, for any conformal map $f$ from $D$ into $\hat{\mathbf{C}}$.

We may now prove the following

THEOREM. Let $D$ be a conformally rigid domain, and $\Gamma=\hat{\mathbf{C}} \backslash D$. Then $m(\Gamma)$ $=0$.

ProOF. We may without loss of generality assume that $\infty \in D$. We shall assume that $m(\Gamma)>0$, and conclude that $D$ is not conformally rigid.

Since $\Gamma$ is compact with $m(\Gamma)>0$, Nguyen's theorem gives a nonconstant bounded holomorphic Lipschitz function $F$ on $D$. For convenience, we write

$$
G=F^{\prime} F^{\prime \prime \prime}-\frac{3}{2} F^{\prime \prime^{2}}
$$

Further, let $A=\operatorname{Lip}(F)$. Now put

$$
f(z)=z+\varepsilon F(z)
$$

with $|\varepsilon|<1 / A$. Then $f$ is conformal on $D$. Note that $f$ is not a Möbius transformation unless $\varepsilon=0$. We have

$$
S(f)=\left(\varepsilon F^{\prime \prime \prime}+\varepsilon^{2} G\right) /\left(1+\varepsilon F^{\prime}\right)^{2}
$$

Now since $F$ satisfies a Lipschitz condition, the inequality $\left|F^{\prime}(z)\right| \leq A$ is valid in $D$. Furthermore, we have

$$
|S(f, z)| \leq 12 \rho_{D}(z)^{2}
$$

in $D$ by the theorem of Beardon and Gehring. Thus

$$
\left|\varepsilon F^{\prime \prime \prime}(z)+\varepsilon^{2} G(z)\right| \leq\left|1+\varepsilon F^{\prime}(z)\right|^{2} 12 \rho_{D}(z)^{2} \leq 12(1+A|\varepsilon|)^{2} \rho_{D}(z)^{2}
$$

in $D$. Putting $\varepsilon= \pm 1 /(2 A)$ gives

$$
\left| \pm F^{\prime \prime \prime}(z) / 2 A+G(z) / 4 A^{2}\right| \leq 27 \rho_{D}(z)^{2}
$$

and this yields

$$
\left|F^{\prime \prime \prime}(z)\right| \leq 54 A \rho_{D}(z)^{2} \quad \text { and } \quad|G(z)| \leq 108 A^{2} \rho_{D}(z)^{2}
$$

by the triangle inequality. But then

$$
\begin{aligned}
|S(f, z)| \rho_{D}(z)^{-2} & \leq \frac{|\varepsilon|\left|F^{\prime \prime \prime}(z)\right|+|\varepsilon|^{2}|G(z)|}{\left(1-|\varepsilon|\left|F^{\prime}(z)\right|\right)^{2}} \rho_{D}(z)^{-2} \\
& \leq \frac{|\varepsilon| 54 A \rho_{D}(z)^{2}+|\varepsilon|^{2} 108 A^{2} \rho_{D}(z)^{2}}{(1-|\varepsilon| A)^{2}} \rho_{D}(z)^{-2} \\
& =\frac{54 A|\varepsilon|+108 A^{2}|\varepsilon|^{2}}{(1-A|\varepsilon|)^{2}}
\end{aligned}
$$


and so

$$
\|S(f)\|_{D}=\sup _{z \in D}|S(f, z)| \rho_{D}(z)^{-2} \leq \frac{54 A|\varepsilon|+108 A^{2}|\varepsilon|^{2}}{(1-A|\varepsilon|)^{2}} .
$$

In particular, $\|S(f)\|_{D}$ tends to zero as $\varepsilon$ tends to zero, and thus $D$ is not a rigid domain.

3. Remarks. From the above, we see that if $S(f)$ is an isolated point of the set of Schwarzians of conformal maps $f: \mathbf{D} \rightarrow \hat{\mathbf{C}}$, in the topology induced by the norm \|\|$_{\mathbf{D}}$, then the omitted set of $f$ has zero area. For the details of the connection between rigid domains and isolated Schwarzians, see [1 1 or 5 ].

The device of using Nguyen's theorem to produce a conformal map was used by Hamilton in [3] to identify the extreme points of an important class of univalent functions.

\section{BIBLIOGRAPHY}

1. K. Astala, Selfsimilar zippers, Proceedings of the workshop on Holomorphic Functions and Moduli, MSRI, Berkeley, Calif., 1987.

2. A. F. Beardon and F. W. Gehring, Schwarzian derivatives, the Poincaré metric and the kernel function, Comment. Math. Helv. 55 (1980), 50-64.

3. D. H. Hamilton, The extreme points of $\Sigma$, Proc. Amer. Math. Soc. 85 (1982), 393-396.

4. Nguyen Xuan Uy, Removable sets of analytic functions satisfying a Lipschitz condition, Ark. Mat. 17 (1979), 19-27.

5. William P. Thurston, Zippers and univalent functions, The Bieberbach Conjecture: Proc. Sympos. on the Occasion of the Proof, Math. Surveys Monos., no. 21, Amer. Math. Soc., Providence, R.I., 1986, pp. 185-197.

Department of Mathematics, University of Michigan, ANN ARbor, Michigan 48109

Current address: Department of Mathematics, Indiana University, Bloomington, Indiana 47405 